\title{
Artificial Lodging: An Effort to Identify a Critical Crop Growth Stage Susceptible to Lodging Leading to Yield Loss and Modification of Yield Structure in Dicoccum Wheat
}

\author{
Sujatha M. Guled*, B. N. Aravinda Kumar, B.T. Nadagouda, \\ Ramesh Babu and C.S. Hunshal
}

Department of Agronomy, University of Agricultural Sciences, Dharwad-580 005, Karnataka, India

*Corresponding author

\begin{tabular}{|c|c|}
\hline & A B S T R A C T \\
\hline & \multirow{5}{*}{$\begin{array}{l}\text { A field experiment was conducted to identify a critical crop growth stage susceptible to } \\
\text { artificial lodging leading to yield loss and yield structure modification in dicoccum wheat } \\
\text { variety 'Mudhol local' during rabi seasons of } 2009 \text { and } 2010 \text { at Agricultural Research } \\
\text { Station, Madhurakhandi, UAS Dharwad, Karnataka. The experiment was laid out in a split } \\
\text { plot design with main plot having two degrees of artificial lodging }\left(45^{\circ} \text { and } 90^{0}\right) \text { and sub- } \\
\text { plots imposed with artificial lodging at four feekes growth stages (FS } 10.3 \text {, FS } 10.52 \text {, FS } \\
11.3 \text { and FS 11.52) and a control (without artificial lodging) and the treatment } \\
\text { combinations were replicated thrice. Between degrees of artificial lodging, } 45^{0} \text { recorded } \\
\text { significantly higher grain yield of } 1987 \mathrm{~kg} \mathrm{ha}^{-1} \text { compared to } 90^{0}\left(1796 \mathrm{~kg} \mathrm{ha}^{-1}\right) \text {. Among } \\
\text { different stages of lodging, before-heading stage being on par with non-lodged } \\
\left.\text { conventional treatment (2082 } \mathrm{kg} \mathrm{ha}^{-1}\right) \text { produced significantly higher grain yield (2201 kg } \\
\left.\mathrm{ha}^{-1}\right) \text {. Lodging at mealy-ripe stage resulted in significantly lower grain yield of } 1681 \mathrm{~kg} \\
\mathrm{ha}^{-1} \text {. Interactions between degrees of lodging and stages of lodging showed that the grain } \\
\text { yield with } 45^{0} \text { compared to } 90^{0} \text { artificial lodging was significantly higher at before-heading } \\
\text { stage }\left(2385 \mathrm{~kg} \mathrm{ha}^{-1}\right) \text {, at hard-kennel stage }\left(1987 \mathrm{~kg}^{-1}\right) \text { and at mealy-ripe stage }(1724 \mathrm{~kg} \\
\left.\mathrm{ha}^{-1}\right) \text { and numerically higher at heading }\left(2061 \mathrm{~kg} \mathrm{ha}^{-1}\right) \text { and in conventional treatment }(2082 \\
\left.\mathrm{kg} \mathrm{ha}^{-1}\right) \text {. }\end{array}$} \\
\hline & \\
\hline Article Info & \\
\hline & \\
\hline & \\
\hline
\end{tabular}

\section{Introduction}

Lodging arising from permanent displacement of plant shoots from upright stature is a complex phenomenon that interferes with photosynthate transportation and dry matter accumulation. It reduces photosynthetic potential, provides a favourable environment for fungal growth and leaf disease development and thus increases agronomic costs and cause yield loss (Fischer and Stapper, 1987). Lodging is the process by which the shoots of small grained cereals are permanently displaced from their vertical stance (Berry et al., 2004). There are two ways in which a cereal plant may lodge either through buckling of the stem (stem lodging) or through failure of the root system (root 
lodging). It can reduce grain yield by $80 \%$ and significantly decrease bread making quality (Pumphrey and Rubenthaler, 1983 and Foulkes et al., 2011). Lodging usually occurs at the early grain filling stage and is influenced by many factors like, frequent strong winds and rain storm, abundant supply of nutrients and water and high planting densities, flat planting and flood irrigation, topography, soil type, previous crop husbandry and diseases, lack of cultivars with lodging resistance under high input conditions (Liu et al., 2003).

Grain filling stage of wheat crop comprises four important feekes stage viz., 90 per cent heading (feekes stage 10.3 - half of heading process completed), flowering (feekes stage 10.52), mealy ripe, contents of kernel soft but dry/ soft dough stage (feekes stage 11.3) and kernel hard/ hard dough stage, ripe for cutting straw dead (feekes stage 11.52). Lodging may occur in any of these four phases of grain filling. The aim of the experimentation was to identify the most susceptible stage for lodging leading to considerable yield and quality loss in dicoccum wheat. Hence, artificial lodging was imposed in the above four phases of grain filling. Angle of lodging also assumes significance in reducing photosynthesis and formation of photosynthates and their transportation from source to sink. Keeping this in view, the crop was imposed with $45^{0}$ and $90^{\circ}$ of artificial lodging in the above mentioned four phases of grain filling.

\section{Materials and Methods}

A field experiment was conducted at the Agricultural Research station, Madhurakhandi of Jamakhandi taluk of Bagalkot district (Latitude $16^{0} 30^{\prime} 18.2^{\prime}$ N, Longitude $75^{\circ} 13^{\prime}$ 52.5" E) in 2008-2009 and 2009-2010 rabi cropping seasons. The soil was a very deep, moderately well drained calcarious cracking vertisol with mean nutrient contents of $130 \mathrm{~kg}$ $\mathrm{ha}^{-1}$ available $\mathrm{N}, 23 \mathrm{~kg} \mathrm{ha}{ }^{-1}$ available phosphorous, $320 \mathrm{~kg} \mathrm{ha}^{-1}$ available potassium at 0 to $30 \mathrm{~cm}$ upper soil layer. The crop was grown in the agro-ecological sub-region (3.3) which is classified as hot-arid ecosystem characterized by prevalence of cool winter, abundant solar resources and often hot and dry winds before maturity.

The wheat crop with Mudhol local variety was sown on 25.11.2008 during the first year and 1.10.2009 during second year. The seed rate $150 \mathrm{~kg} \mathrm{ha}^{-1}$ with $20 \mathrm{~cm}$ row spacing and $10 \mathrm{~cm}$ intra row spacing was followed. The recommended dose of fertilizer was applied with 60:30:20 NPK kg ha' ${ }^{-1}$ and one ton ha ${ }^{-1}$ poultry manure was incorporated fifteen days before sowing. The crop received an average rain fall of $384.3 \mathrm{~mm}$ with 35 rainy days during first year (2008-09) and $799.3 \mathrm{~mm}$ with 55 rainy days during second year (2009-2010). The maximum temperature of $33.19^{\circ} \mathrm{c}$ and $35.16^{\circ} \mathrm{c}$ in March and a minimum temperature of $14.06^{\circ} \mathrm{c}$ in January 2009 and $10.32^{\circ} \mathrm{c}$ in December of 2010 were recorded. The monthly mean relative humidity of $93.5 \%$ at 8.30 am and $72.4 \%$ at $5.30 \mathrm{pm}$ was recorded in September in the first year (2008-09) and the same trend was observed in the second year (2009-10). The lowest monthly mean relative humidity at 8.30 am was recorded in February $(71.7 \%)$ but at $5.30 \mathrm{pm}$ it was $32.8 \%$ in the month of April in the first year (200809). Similar pattern was recorded during second year (2009-10).

The experiment was laid out in a split plot design. The main plots having two degrees of artificial lodging $\left(45^{\circ}\right.$ and $\left.90^{\circ}\right)$ and sub plots were imposed with artificial lodging at four different phases of reproductive stage viz., 90 per cent heading (10.3 feekes stage) half of heading process completed, 50 per cent flowering (10.52 feekes stage) flowering complete to top of ear, soft dough stage (11.3 feekes stage) mealy ripe contents of kernel 
soft but dry, hard kernel / hard dough stage (11.52 feekes stage) where difficult to divide by thumb nail and conventional practice (no lodging). Treatments were replicated thrice. The gross and net plot sizes were $22.5 \mathrm{~m}^{2}$ and $15.3 \mathrm{~m}^{2}$ respectively.

Irrigations adjusted for rainfall, were provided at tillering, at stem elongation and after anthesis in both the cropping seasons. The remaining cultural operations viz., weeding, inter cultivation and plant protection measures were taken timely to keep the crop free from weeds, pests and diseases and moisture stress. The crop was harvested on 26.03.2009 and 12.3.2010 during first year and second year, respectively.

\section{Results and Discussion}

\section{Effect on growth parameters}

The plant height with $45^{\circ}(86.80 \mathrm{~cm})$ compared to $90^{\circ}$ of lodging $(82.790 \mathrm{~m})$ at hard kernel stage recorded significantly greater plant height $(91.43 \mathrm{~cm})$ compared to at heading $(82.76 \mathrm{~cm})$, before heading $(84.11 \mathrm{~cm})$ and soft dough stage $(88.91 \mathrm{~cm})$, respectively. The similar pattern of significant superiority was observed with respect to stem length and peduncle length (Table 1). The significant values of plant height, stem length and peduncle length at hard kernel stage with $45^{0}$ of artificial lodging is quite obvious, because the crop was not put to any stress due to lodging up to hard kernel stage. The lesser plant height, stem length and peduncle length in other stages may be attributed to retardation of extension growth due to lower or supraoptimal auxin levels or lower gibberellin levels (Biddington, 1986) among stages of artificial lodging.

The total number of tillers, productive tillers per meter row length as well as per square meter area was significantly higher with $45^{\circ}$ compared to $90^{\circ}$ of artificial lodging at all the stages of lodging (Table 2). Further, the number of tillers (92.5), productive tillers (82.0) per meter row length and per square meter area were significantly higher with before heading stage of crop growth (371.25 and 322.92, respectively). On the contrary, the number of tillers (80.5), productive tillers (68.25) per meter row length and per square meter area (305.50 and 273.34, respectively) were significantly lower with $45^{\circ}$ of lodging at mealy ripe stage. The interaction effects occurring between stages of lodging and degrees of lodging conspicuously indicated that the number of tillers, productive tillers per meter row length (97.5 and 89.5) and per square meter area (391.5 and 338.67) were significantly higher in before heading stage with $45^{\circ}$ of artificial lodging compared to rest of the stages with $45^{\circ}$ of artificial lodging. The higher number of tillers and productive tillers per meter row length and per square meter area may be attributed to production of ethylene on artificial lodging (Biddington, 1986). Ethylene is known to reduce apical dominance, increase tillering, cytokines and ABA (Davies and Zhang, 1991), (Zahedi and Jenner 2003; Davies 2004); Mitocondria, Golgi-bodies and Mycoplasts in root tip cells (Yang, 2011). This change is known to improve significantly ultra structure of root tip cells, bringing in conspicuously greater cross sectional area in the effective root zone with higher root oxidation activity (ROA) enabling the plant to have greater access to nutrients to roots together root sourced hormones are transported via transpiration stream to the above ground parts where they regulate growth and development (Yang et al., 2012). The number of these organelles was significantly correlated with tiller number at tillering stage. It might be due to maintenance of optimum to higher leaf area index (Table 3 ), leaf area duration (Table 4), higher SPAD meter reading (greenness of flag leaf) (Table 5), light interception (Table 6) throughout the 
crop growth. It is important for obtaining higher photosynthetic efficiency, which in turn help to produce more dry matter (Table 7) and ultimately increase the grain yield. Artificial lodging with $45^{\circ}$ compared to $90^{\circ}$ lodging recorded significantly higher LAI (2.76 at boot leaf, 3.25 at initial flowering and
2.55 at mealy ripe stage), LAD (78.13 days at mealy ripe, 75.4 days at harvest), light interception $(28.67 \%$ at boot leaf, $42.27 \%$ at initial flowering and $33.63 \%$ at mealy ripe stage), higher SPAD meter reading (35.94\% at boot leaf $37.11 \%$ at initial flowering and $22.10 \%$ at mealy ripe stage).

Table.1 Influence of degree of bending at different stages of crop growth on plant height, stem length, peduncle length

\begin{tabular}{|c|c|c|c|c|c|c|c|c|c|}
\hline \multirow{3}{*}{$\begin{array}{l}\text { Treatment of } \\
\text { bending }\end{array}$} & \multicolumn{9}{|c|}{ Pooled (over two years) } \\
\hline & \multicolumn{3}{|c|}{ Plant height (cm) } & \multicolumn{3}{|c|}{ Stem length (cm) } & \multicolumn{3}{|c|}{ Peduncle length $(\mathrm{cm})$} \\
\hline & $45^{0}$ & $90^{\circ}$ & Mean & $45^{0}$ & $90^{0}$ & Mean & $45^{0}$ & $90^{0}$ & Mean \\
\hline Before heading & 84.11 & 81.09 & 82.60 & 60.71 & 52.24 & 56.47 & 30.63 & 28.17 & 29.40 \\
\hline At heading & 82.76 & 80.69 & 81.72 & 56.88 & 56.47 & 56.67 & 27.31 & 24.74 & 26.03 \\
\hline At mealy stage & 88.91 & 79.11 & 84.01 & 69.45 & 57.19 & 63.32 & 31.86 & 30.06 & 30.96 \\
\hline $\begin{array}{l}\text { At hard kernel } \\
\text { stage }\end{array}$ & 91.43 & 90.24 & 90.84 & 72.83 & 70.86 & 71.85 & 33.15 & 30.31 & 31.73 \\
\hline Conventional & - & - & 91.69 & - & - & 72.64 & - & - & 32.16 \\
\hline Overall Mean & 86.80 & 82.79 & 86.17 & 64.97 & 59.19 & 64.19 & 30.74 & 28.32 & 30.05 \\
\hline $\begin{array}{l}\text { Comparing } \\
\text { means of }\end{array}$ & $\begin{array}{l}\text { S.Em } \\
\quad \pm\end{array}$ & \multicolumn{2}{|c|}{ C.D. at $5 \%$} & $\begin{array}{l}\text { S.Em. } \\
\quad \pm\end{array}$ & \multicolumn{2}{|c|}{ C.D. at $5 \%$} & $\begin{array}{l}\text { S.Em. } \\
\quad \pm\end{array}$ & \multicolumn{2}{|c|}{ C.D. at $5 \%$} \\
\hline Season & 1.17 & 5.02 & - & 0.95 & 4.10 & - & 0.67 & 2.89 & - \\
\hline $\begin{array}{l}\text { Degree of } \\
\text { bending }\end{array}$ & 0.70 & 1.43 & - & 1.00 & 2.03 & - & 0.50 & 1.00 & - \\
\hline Stages of bending & 1.11 & 2.26 & - & 1.59 & 3.22 & - & 0.78 & 1.59 & - \\
\hline \multicolumn{10}{|c|}{ Interactions between: } \\
\hline $\begin{array}{l}\text { Degrees of } \\
\text { bending at a } \\
\text { given stage or } \\
\text { different stages of } \\
\text { bending for a } \\
\text { given season or } \\
\text { over seasons } \\
\end{array}$ & 1.00 & 2.02 & - & 1.42 & 2.88 & - & 0.70 & 1.42 & - \\
\hline $\begin{array}{l}\text { Stages of bending } \\
\text { for a given degree } \\
\text { of bending or } \\
\text { different degrees } \\
\text { of bending for a } \\
\text { given season or } \\
\text { over seasons }\end{array}$ & 1.58 & 3.19 & - & 2.24 & 4.55 & - & 1.11 & 2.24 & - \\
\hline
\end{tabular}


Table.2 Influence of degree of bending at different stages of crop growth on number of tillers and number of productive tillers at harvest

\begin{tabular}{|c|c|c|c|c|c|c|c|c|c|c|c|c|}
\hline \multirow{3}{*}{$\begin{array}{l}\text { Treatment of } \\
\text { bending }\end{array}$} & \multicolumn{12}{|c|}{ Pooled (over two years) } \\
\hline & \multicolumn{3}{|c|}{$\begin{array}{l}\text { No. of Productive } \\
\text { Tillers } \mathbf{m}^{-1} \text { row } \\
\text { length }\end{array}$} & \multicolumn{3}{|c|}{$\begin{array}{l}\text { No. of Tillers } \mathbf{~ m}^{-1} \\
\text { row length }\end{array}$} & \multicolumn{3}{|c|}{$\begin{array}{c}\text { No. of Productive } \\
\text { Tillers } \mathrm{m}^{-2}\end{array}$} & \multicolumn{3}{|c|}{ No. of Tillers $\mathbf{m}^{-2}$} \\
\hline & $45^{\circ}$ & $90^{\circ}$ & Mean & $45^{0}$ & $90^{0}$ & Mean & $45^{0}$ & $90^{\circ}$ & Mean & $45^{\circ}$ & $90^{0}$ & Mean \\
\hline Before heading & 89.50 & 74.50 & 82.00 & 97.50 & 87.50 & 92.50 & 338.67 & 307.17 & 322.92 & 391.50 & 351.00 & 371.25 \\
\hline At heading & 80.00 & 73.59 & 76.79 & 86.75 & 84.50 & 85.63 & 301.33 & 291.84 & 296.59 & 363.50 & 331.17 & 347.33 \\
\hline At mealy stage & 68.25 & 55.00 & 61.63 & 80.50 & 72.50 & 76.50 & 273.34 & 218.34 & 245.83 & 305.50 & 276.50 & 291.00 \\
\hline At hard kernel stage & 72.75 & 67.50 & 70.13 & 81.00 & 80.50 & 80.75 & 294.86 & 284.34 & 289.60 & 332.50 & 314.00 & 323.25 \\
\hline Conventional & - & - & 78.88 & - & - & 91.86 & - & - & 305.27 & - & - & 370.26 \\
\hline Overall Mean & 77.63 & 67.65 & 73.89 & 86.44 & 81.25 & 85.45 & 302.05 & 275.42 & 292.04 & 348.25 & 318.17 & 340.62 \\
\hline Comparing means of & $\begin{array}{c}\text { S.Em. } \\
\pm\end{array}$ & \multicolumn{2}{|c|}{ C.D. at $5 \%$} & $\begin{array}{c}\text { S.Em } \\
. \pm\end{array}$ & \multicolumn{2}{|c|}{ C.D. at $5 \%$} & S.Em. \pm & \multicolumn{2}{|c|}{ C.D. at $5 \%$} & $\begin{array}{c}\text { S.Em. } \\
\pm\end{array}$ & \multicolumn{2}{|c|}{ C.D. at $5 \%$} \\
\hline Degree of bending & 1.26 & 4.10 & - & 1.29 & 3.61 & - & 4.34 & 13.02 & - & 5.83 & 17.50 & - \\
\hline Stages of bending & 1.99 & 5.04 & - & 2.03 & 4.12 & - & 6.86 & 20.58 & - & 9.21 & 18.68 & - \\
\hline \multicolumn{13}{|l|}{ Interactions between: } \\
\hline $\begin{array}{l}\text { Degrees of bending at } \\
\text { a given stage or } \\
\text { different stages of } \\
\text { bending for a given } \\
\text { season or over } \\
\text { seasons }\end{array}$ & 1.78 & 5.34 & - & 1.82 & 5.46 & - & 6.14 & 19.96 & & 8.24 & 24.72 & - \\
\hline $\begin{array}{l}\text { Stages of bending for } \\
\text { a given degree of } \\
\text { bending or different } \\
\text { degrees of bending } \\
\text { for a given season or } \\
\text { over seasons }\end{array}$ & 2.82 & 5.71 & - & 2.87 & 5.83 & - & 6.70 & 19.67 & & 6.03 & 18.09 & - \\
\hline
\end{tabular}


Table.3 Influence of degree of bending on leaf area index at different stages of crop growth

\begin{tabular}{|c|c|c|c|c|c|c|c|c|c|}
\hline \multirow[t]{3}{*}{ Treatment of bending } & \multicolumn{9}{|c|}{ Pooled (over two years) } \\
\hline & \multicolumn{3}{|c|}{ Boot leaf stage } & \multicolumn{3}{|c|}{ Initial flowering } & \multicolumn{3}{|c|}{ Mealy ripe } \\
\hline & $45^{0}$ & $90^{0}$ & Mean & $45^{0}$ & $90^{0}$ & Mean & $45^{0}$ & $90^{0}$ & Mean \\
\hline Before heading & 2.76 & 2.31 & 2.54 & 3.25 & 3.09 & 3.17 & 2.55 & 2.34 & 2.45 \\
\hline At heading & 2.51 & 2.22 & 2.37 & 3.14 & 2.83 & 2.98 & 1.13 & 0.92 & 1.03 \\
\hline At mealy stage & 2.58 & 2.49 & 2.54 & 3.14 & 3.12 & 3.13 & 2.12 & 1.71 & 1.91 \\
\hline At hard kernel stage & 2.57 & 2.55 & 2.56 & 3.12 & 3.09 & 3.11 & 2.32 & 1.91 & 2.11 \\
\hline Conventional & - & - & 2.51 & - & - & 3.12 & - & - & 1.13 \\
\hline Overall Mean & 2.61 & 2.40 & 2.50 & 3.17 & 3.03 & 3.10 & 2.03 & 1.73 & 1.88 \\
\hline Comparing means of & $\begin{array}{c}\text { S.Em. } \\
\pm\end{array}$ & \multicolumn{2}{|c|}{ C.D. at $5 \%$} & $\begin{array}{c}\text { S.Em. } \\
\pm\end{array}$ & \multicolumn{2}{|c|}{ C.D. at $5 \%$} & $\begin{array}{c}\text { S.Em. } \\
\pm\end{array}$ & \multicolumn{2}{|c|}{ C.D. at $5 \%$} \\
\hline Season & 0.03 & 0.09 & - & 0.11 & 0.33 & & 0.07 & 0.21 & - \\
\hline Degree of lodging & 0.06 & 0.18 & - & 0.12 & 0.36 & & 0.05 & 0.15 & - \\
\hline Stages of bending & 0.10 & 0.3 & - & 0.19 & 0.57 & & 0.08 & 0.24 & - \\
\hline \multicolumn{10}{|l|}{ Interactions between: } \\
\hline $\begin{array}{l}\text { Degrees of bending at a } \\
\text { given stage or different } \\
\text { stages of bending for a } \\
\text { given season or over } \\
\text { seasons }\end{array}$ & 0.09 & 0.27 & - & 0.17 & 0.51 & & 0.07 & 0.21 & - \\
\hline $\begin{array}{l}\text { Stages of bending for a } \\
\text { given degree of bending or } \\
\text { different degrees of } \\
\text { bending for a given season } \\
\text { or over seasons }\end{array}$ & 0.14 & 0.42 & - & 0.27 & 0.81 & & 0.11 & 0.33 & - \\
\hline
\end{tabular}

Table.4 Influence of degree of bending on leaf area duration at different stages of crop growth

\begin{tabular}{|c|c|c|c|c|c|c|}
\hline \multirow[t]{3}{*}{ Treatment of bending } & \multicolumn{6}{|c|}{ Pooled (over two years) } \\
\hline & \multicolumn{3}{|c|}{ Mealy ripe } & \multicolumn{3}{|c|}{ Harvest } \\
\hline & $45^{0}$ & $90^{0}$ & Mean & $45^{0}$ & $90^{0}$ & Mean \\
\hline Before heading & 78.13 & 54.00 & 66.07 & 75.40 & 54.35 & 64.88 \\
\hline At heading & 73.52 & 50.45 & 61.99 & 55.51 & 37.4 & 46.46 \\
\hline At mealy stage & 74.43 & 56.00 & 65.22 & 68.38 & 48.25 & 58.32 \\
\hline At hard kernel stage & 73.97 & 56.45 & 65.22 & 70.72 & 50.00 & 60.36 \\
\hline Conventional & - & - & 73.19 & - & - & 55.25 \\
\hline Overall Mean & 75.01 & 54.23 & 64.63 & 67.50 & 47.50 & 57.50 \\
\hline Comparing means of & S.Em. \pm & \multicolumn{2}{|c|}{ C.D. at $5 \%$} & S.Em. \pm & \multicolumn{2}{|c|}{ C.D. at $5 \%$} \\
\hline Season & 0.48 & 2.05 & - & 8.24 & 35.44 & - \\
\hline Degree of lodging & 2.12 & 4.30 & - & 3.20 & 6.50 & - \\
\hline Stages of bending & 3.35 & 6.79 & - & 5.06 & 10.27 & - \\
\hline \multicolumn{7}{|l|}{ Interactions between: } \\
\hline $\begin{array}{l}\text { Degrees of bending at a given stage } \\
\text { or different stages of bending for a } \\
\text { given season or over seasons }\end{array}$ & 3.00 & 6.08 & - & 4.53 & 9.18 & - \\
\hline $\begin{array}{l}\text { Stages of bending for a given } \\
\text { degree of bending or different } \\
\text { degrees of bending for a given } \\
\text { season or over seasons }\end{array}$ & 4.74 & 9.61 & - & 7.16 & 14.52 & - \\
\hline
\end{tabular}


Table.5 Influence of degree of bending at different stages of crop growth on spad meter reading

\begin{tabular}{|c|c|c|c|c|c|c|c|c|c|}
\hline \multirow{3}{*}{$\begin{array}{l}\text { Treatment of } \\
\text { bending }\end{array}$} & \multicolumn{9}{|c|}{ Pooled (2008-09 and 2009-10) } \\
\hline & \multicolumn{3}{|c|}{ Boot leaf stage } & \multicolumn{3}{|c|}{ Initial flowering } & \multicolumn{3}{|c|}{ Mealy ripe } \\
\hline & $45^{0}$ & $90^{0}$ & Mean & $45^{0}$ & $90^{0}$ & Mean & $45^{0}$ & $90^{\circ}$ & Mean \\
\hline Before heading & 35.94 & 31.31 & 33.63 & 37.11 & 32.90 & 35.00 & 22.10 & 18.19 & 20.14 \\
\hline At heading & 34.66 & 32.40 & 33.53 & 35.28 & 32.32 & 33.80 & 22.46 & 18.68 & 20.57 \\
\hline At mealy stage & 34.82 & 34.37 & 34.60 & 35.79 & 35.47 & 35.63 & 18.71 & 16.34 & 17.53 \\
\hline $\begin{array}{l}\text { At hard kernel } \\
\text { stage }\end{array}$ & 34.36 & 33.94 & 34.15 & 34.19 & 32.51 & 33.35 & 18.81 & 15.88 & 17.35 \\
\hline Conventional & - & - & 33.42 & - & - & 34.46 & - & - & 22.95 \\
\hline Overall Mean & 34.94 & 33.00 & 33.98 & 35.59 & 33.30 & 34.45 & 20.52 & 17.27 & 19.71 \\
\hline $\begin{array}{l}\text { Comparing means } \\
\text { of }\end{array}$ & $\begin{array}{l}\text { S.Em. } \\
\quad \pm\end{array}$ & \multicolumn{2}{|c|}{ C.D. at $5 \%$} & S.Em. \pm & \multicolumn{2}{|c|}{ C.D. at $5 \%$} & S.Em. \pm & \multicolumn{2}{|c|}{ C.D. at $5 \%$} \\
\hline Season & 0.08 & 0.34 & - & 0.81 & 3.48 & - & 0.48 & 2.07 & - \\
\hline Degree of lodging & 0.28 & 0.58 & - & 0.51 & 1.03 & - & 0.32 & 0.66 & - \\
\hline Stages of bending & 0.45 & 0.91 & - & 0.81 & 1.63 & - & 0.51 & 1.04 & - \\
\hline \multicolumn{10}{|c|}{ Interactions between: } \\
\hline $\begin{array}{l}\text { Degrees of bending } \\
\text { at a given stage or } \\
\text { different stages of } \\
\text { bending for a given } \\
\text { season or over } \\
\text { seasons }\end{array}$ & 0.40 & 0.81 & - & 0.72 & 1.46 & - & 0.46 & 0.93 & - \\
\hline $\begin{array}{l}\text { stages of bending } \\
\text { for a given degree } \\
\text { of bending or } \\
\text { different degrees of } \\
\text { bending for a given } \\
\text { season or over } \\
\text { seasons }\end{array}$ & 0.63 & 1.29 & - & 1.14 & 2.31 & - & 0.72 & 1.47 & - \\
\hline
\end{tabular}


Table.6 Influence of degree of bending at different stages of crop growth on light interception $(\%)$

\begin{tabular}{|c|c|c|c|c|c|c|c|c|c|}
\hline \multirow{3}{*}{$\begin{array}{l}\text { Treatment of } \\
\text { bending }\end{array}$} & \multicolumn{9}{|c|}{ Pooled (2008-09 and 2009-10) } \\
\hline & \multicolumn{3}{|c|}{ Boot leaf stage } & \multicolumn{3}{|c|}{ Initial flowering } & \multicolumn{3}{|c|}{ Mealy ripe } \\
\hline & $45^{0}$ & $90^{0}$ & Mean & $45^{0}$ & $90^{0}$ & $\begin{array}{c}\text { Mea } \\
\text { n }\end{array}$ & $45^{0}$ & $90^{\circ}$ & Mean \\
\hline Before heading & 28.67 & $\begin{array}{c}27.7 \\
0\end{array}$ & 28.18 & 42.27 & $\begin{array}{c}37.9 \\
3\end{array}$ & 40.10 & 33.63 & 28.79 & 31.21 \\
\hline At heading & 27.78 & $\begin{array}{c}27.2 \\
1\end{array}$ & 27.50 & 37.84 & $\begin{array}{c}35.4 \\
9\end{array}$ & 31.85 & 30.95 & 24.95 & 27.95 \\
\hline At mealy stage & 28.03 & $\begin{array}{c}27.5 \\
5\end{array}$ & 27.80 & 41.03 & $\begin{array}{c}40.4 \\
0\end{array}$ & 40.71 & 24.63 & 23.24 & 23.93 \\
\hline At hard kernel stage & 27.58 & $\begin{array}{c}27.4 \\
5\end{array}$ & 27.52 & 27.92 & $\begin{array}{c}27.4 \\
7\end{array}$ & 27.70 & 27.56 & 23.68 & 25.62 \\
\hline Conventional & - & - & 27.59 & - & - & 39.79 & - & - & 30.58 \\
\hline Overall Mean & 28.02 & $\begin{array}{c}27.4 \\
8\end{array}$ & 27.75 & 37.27 & $\begin{array}{c}35.3 \\
2\end{array}$ & 36.29 & 29.19 & 25.17 & 27.86 \\
\hline Comparing means of & $\begin{array}{c}\text { S.Em. } \\
\pm\end{array}$ & \multicolumn{2}{|c|}{ C.D. at $5 \%$} & $\begin{array}{c}\text { S.Em. } \\
\pm\end{array}$ & \multicolumn{2}{|c|}{ C.D. at $5 \%$} & $\begin{array}{c}\text { S.Em. } \\
\pm\end{array}$ & \multicolumn{2}{|c|}{ C.D. at $5 \%$} \\
\hline Season & 0.61 & 2.64 & - & 0.25 & 1.08 & - & 0.36 & 1.54 & - \\
\hline Degree of lodging & 0.42 & 0.86 & - & 0.24 & 0.48 & - & 0.83 & 1.69 & - \\
\hline Stages of bending & 0.67 & 1.37 & - & 0.37 & 0.76 & - & 1.31 & 2.67 & - \\
\hline \multicolumn{10}{|l|}{ Interactions between: } \\
\hline $\begin{array}{l}\text { Degrees of bending } \\
\text { at a given stage or } \\
\text { different stages of } \\
\text { bending for a given } \\
\text { season or over } \\
\text { seasons }\end{array}$ & 0.60 & 1.22 & - & 0.33 & 0.68 & & 1.18 & 2.38 & - \\
\hline $\begin{array}{l}\text { Stages of bending } \\
\text { for a given degree of } \\
\text { bending or different } \\
\text { degrees of bending } \\
\text { for a given season or } \\
\text { over seasons }\end{array}$ & 0.95 & 1.93 & - & 0.53 & 1.07 & & 1.86 & 3.77 & - \\
\hline
\end{tabular}


Table.7 Influence of degree of bending at different stages of crop growth on total dry matter accumulation $\left(\mathrm{g}\right.$ plant $\left.{ }^{-1}\right)$

\begin{tabular}{|c|c|c|c|c|c|c|c|c|c|c|c|c|}
\hline \multirow[t]{3}{*}{ Treatment of bending } & \multicolumn{12}{|c|}{ Pooled (2008-09 and 2009-10) } \\
\hline & \multicolumn{3}{|c|}{ Boot leaf stage } & \multicolumn{3}{|c|}{ Initial flowering } & \multicolumn{3}{|c|}{ Mealy ripe } & \multicolumn{3}{|c|}{ Harvest } \\
\hline & $45^{0}$ & $90^{0}$ & Mean & $45^{0}$ & $90^{0}$ & Mean & $45^{0}$ & $90^{0}$ & Mean & $45^{0}$ & $90^{0}$ & Mean \\
\hline Before heading & 50.80 & $\begin{array}{c}48.2 \\
2\end{array}$ & 49.51 & 114.20 & $\begin{array}{c}100.1 \\
7\end{array}$ & 107.18 & 130.16 & $\begin{array}{c}117.5 \\
6\end{array}$ & $\begin{array}{c}123.8 \\
6\end{array}$ & 113.95 & 87.60 & 100.77 \\
\hline At heading & 49.83 & $\begin{array}{c}49.0 \\
5\end{array}$ & 49.44 & 104.19 & 95.44 & 99.81 & 122.43 & $\begin{array}{c}109.8 \\
3\end{array}$ & 116.13 & 97.80 & 85.32 & 91.56 \\
\hline At mealy stage & 49.56 & $\begin{array}{c}49.0 \\
9\end{array}$ & 49.33 & 108.22 & $\begin{array}{c}107.4 \\
3\end{array}$ & 107.83 & 95.48 & 86.53 & 88.51 & 82.07 & 71.74 & 76.91 \\
\hline At hard kernel stage & 47.21 & $\begin{array}{c}46.8 \\
3\end{array}$ & 47.02 & 106.60 & $\begin{array}{c}104.9 \\
5\end{array}$ & 105.78 & 115.58 & $\begin{array}{c}100.7 \\
7\end{array}$ & $\begin{array}{c}108.1 \\
9\end{array}$ & 90.15 & 77.48 & 83.82 \\
\hline Conventional & - & - & 49.89 & - & - & 105.07 & - & - & $\begin{array}{c}121.9 \\
7\end{array}$ & - & - & 102.10 \\
\hline Overall Mean & 49.35 & $\begin{array}{c}48.3 \\
0\end{array}$ & 48.83 & 108.30 & $\begin{array}{c}102.0 \\
0\end{array}$ & 105.15 & 115.44 & $\begin{array}{c}104.1 \\
6\end{array}$ & 112.23 & 96.00 & 80.54 & 91.03 \\
\hline Comparing means of & $\begin{array}{c}\text { S.Em. } \\
\pm\end{array}$ & \multicolumn{2}{|c|}{ C.D. at $5 \%$} & $\begin{array}{c}\text { S.Em. } \\
\pm\end{array}$ & \multicolumn{2}{|c|}{ C.D. at $5 \%$} & $\begin{array}{c}\text { S.Em. } \\
\pm\end{array}$ & \multicolumn{2}{|c|}{ C.D. at $5 \%$} & $\begin{array}{c}\text { S.Em. } \\
\pm\end{array}$ & \multicolumn{2}{|c|}{ C.D. at $5 \%$} \\
\hline Degree of bending & 0.64 & 1.30 & - & 0.70 & 1.43 & - & 2.43 & 4.93 & & 2.54 & 7.62 & - \\
\hline Stages of bending & 1.01 & 2.06 & - & 1.11 & 2.25 & - & 3.84 & 7.79 & & 3.78 & 11.34 & - \\
\hline \multicolumn{13}{|l|}{ Interactions between: } \\
\hline $\begin{array}{l}\text { Degrees of bending at a given } \\
\text { stage or different stages of } \\
\text { bending for a given season or } \\
\text { over seasons }\end{array}$ & 0.91 & 1.84 & - & 0.99 & 2.02 & - & 3.44 & 6.97 & - & 4.57 & 13.71 & - \\
\hline $\begin{array}{l}\text { Stages of bending for a given } \\
\text { degree of bending or different } \\
\text { degrees of bending for a given } \\
\text { season or over seasons }\end{array}$ & 1.44 & 2.91 & - & 1.57 & 3.19 & - & 5.43 & 11.02 & - & 5.70 & 17.10 & - \\
\hline
\end{tabular}


Table.8 Influence of degree of bending at different stages of crop growth on panicle length, number of grains, number of spikelet per panicle at harvest

\begin{tabular}{|c|c|c|c|c|c|c|c|c|c|}
\hline \multirow{3}{*}{$\begin{array}{l}\text { Treatment of } \\
\text { bending }\end{array}$} & \multicolumn{9}{|c|}{ Pooled (2008-09 and 2009-10) } \\
\hline & \multicolumn{3}{|c|}{ Panicle length (cm) } & \multicolumn{3}{|c|}{$\begin{array}{c}\text { Number of grains } \\
\text { panicle }^{-1}\end{array}$} & \multicolumn{3}{|c|}{$\begin{array}{c}\text { Number of spike lets } \\
\text { panicle }^{-1}\end{array}$} \\
\hline & $45^{0}$ & $90^{0}$ & Mean & $45^{\circ}$ & $90^{0}$ & Mean & $45^{0}$ & $90^{0}$ & Mean \\
\hline Before heading & 6.41 & 6.11 & 6.26 & 34.84 & 33.50 & 34.17 & 16.97 & 16.60 & 16.78 \\
\hline At heading & 6.09 & 5.63 & 5.86 & 34.17 & 33.50 & 33.84 & 16.57 & 16.24 & 16.40 \\
\hline At mealy stage & 6.84 & 6.22 & 6.53 & 33.50 & 30.25 & 31.88 & 16.57 & 14.34 & 15.45 \\
\hline $\begin{array}{l}\text { At hard kernel } \\
\text { stage }\end{array}$ & 7.07 & 6.57 & 6.82 & 33.67 & 32.34 & 33.00 & 16.64 & 15.70 & 16.17 \\
\hline Conventional & - & - & 7.13 & - & - & 35.19 & - & - & 18.04 \\
\hline Overall Mean & 6.60 & 6.14 & 6.52 & 34.04 & 32.40 & 33.61 & 16.69 & 15.72 & 16.57 \\
\hline $\begin{array}{l}\text { Comparing } \\
\text { means of }\end{array}$ & $\begin{array}{l}\text { S.Em. } \\
\quad \pm\end{array}$ & \multicolumn{2}{|c|}{ C.D. at $5 \%$} & $\begin{array}{l}\text { S.Em. } \\
\quad \pm\end{array}$ & \multicolumn{2}{|c|}{ C.D. at $5 \%$} & $\begin{array}{l}\text { S.Em. } \\
\quad \pm\end{array}$ & \multicolumn{2}{|c|}{ C.D. at $5 \%$} \\
\hline Season & 0.08 & 0.35 & - & 0.50 & 2.15 & - & 0.46 & 1.98 & - \\
\hline $\begin{array}{ll}\text { Degree } & \text { of } \\
\text { lodging } & \\
\end{array}$ & 0.75 & 1.15 & - & 0.47 & 0.94 & - & 0.39 & 0.80 & - \\
\hline $\begin{array}{ll}\text { Stages } & \text { of } \\
\text { bending } & \\
\end{array}$ & 0.12 & 0.24 & - & 0.74 & 1.49 & - & 0.62 & 1.26 & - \\
\hline \multicolumn{10}{|c|}{ Interactions between: } \\
\hline $\begin{array}{l}\text { Degrees of } \\
\text { bending at a } \\
\text { given stage or } \\
\text { different stages } \\
\text { of bending for a } \\
\text { given season or } \\
\text { over seasons } \\
\end{array}$ & 0.11 & 0.21 & - & 0.66 & 1.34 & - & 0.56 & 1.13 & - \\
\hline $\begin{array}{lr}\text { Stages } & \text { of } \\
\text { bending for } & \text { a } \\
\text { given degree } & \text { of } \\
\text { bending } & \text { or } \\
\text { different } & \\
\text { degrees } & \text { of } \\
\text { bending for } & \text { a } \\
\text { given season } & \text { or } \\
\text { over seasons } & \end{array}$ & 0.17 & 0.34 & - & 1.04 & 2.11 & - & 0.88 & 1.79 & - \\
\hline
\end{tabular}


Table.9 Influence of degree of bending at different stages of crop growth on thousand grain weight, grain yield, straw yield

\begin{tabular}{|c|c|c|c|c|c|c|c|c|c|}
\hline \multirow[t]{3}{*}{ Treatment of bending } & \multicolumn{9}{|c|}{ Pooled (2008-09 and 2009-10) } \\
\hline & \multicolumn{3}{|c|}{$\begin{array}{l}\text { Thousand grain } \\
\text { weight }(\mathrm{g})\end{array}$} & \multicolumn{3}{|c|}{$\begin{array}{c}\text { Grain yield } \\
\left(\mathrm{kg} \mathrm{ha}^{-1}\right)\end{array}$} & \multicolumn{3}{|c|}{$\begin{array}{l}\text { Straw yield } \\
\left(\mathrm{kg} \mathrm{ha}^{-1}\right)\end{array}$} \\
\hline & $45^{0}$ & $90^{\circ}$ & Mean & $45^{0}$ & $90^{\circ}$ & Mean & $45^{0}$ & $90^{0}$ & Mean \\
\hline Before heading & 39.80 & 38.05 & 39.03 & 2385 & 2016 & 2201 & 5057 & 4545 & 4801 \\
\hline At heading & 38.38 & 36.24 & 37.31 & 2061 & 1979 & 2020 & 4745 & 4441 & 4593 \\
\hline At mealy stage & 35.44 & 33.07 & 34.25 & 1724 & 1637 & 1681 & 4208 & 3788 & 3998 \\
\hline At hard kernel stage & 37.23 & 33.82 & 35.53 & 1987 & 1796 & 1891 & 4615 & 4143 & 4379 \\
\hline Conventional & - & - & 39.00 & - & - & 2082 & - & - & 4789 \\
\hline Overall Mean & 37.72 & 35.29 & 37.00 & 2040 & 1857 & 1975 & 4656 & 4230 & 4512 \\
\hline Comparing means of & $\begin{array}{l}\text { S.Em. } \\
\pm\end{array}$ & \multicolumn{2}{|c|}{ C.D. at $5 \%$} & $\begin{array}{l}\text { S.Em. } \\
\pm\end{array}$ & \multicolumn{2}{|c|}{ C.D. at $5 \%$} & $\begin{array}{l}\text { S.Em } \\
\pm\end{array}$ & \multicolumn{2}{|c|}{ C.D. at $5 \%$} \\
\hline Season & 0.39 & 1.68 & - & 0.26 & 1.10 & - & 0.04 & 0.18 & - \\
\hline Degree of lodging & 0.43 & 0.86 & - & 0.39 & 0.80 & - & 0.47 & 0.96 & - \\
\hline Stages of bending & 0.67 & 1.37 & - & 0.62 & 1.26 & - & 0.75 & 1.52 & - \\
\hline \multicolumn{10}{|l|}{ Interactions between: } \\
\hline $\begin{array}{l}\text { Degrees of bending at a given stage } \\
\text { or different stages of bending for a } \\
\text { given season or over seasons }\end{array}$ & 0.60 & 1.22 & - & 0.56 & 1.13 & - & 0.67 & 1.36 & - \\
\hline $\begin{array}{l}\text { stages of bending for a given } \\
\text { degree of bending or differen } \\
\text { degrees of bending for a given } \\
\text { season or over seasons }\end{array}$ & 0.95 & 1.93 & - & 0.88 & 1.78 & - & 1.06 & 2.15 & - \\
\hline
\end{tabular}

The results have clearly indicated that among different stages of lodging, the mealy ripe stage recorded significantly lower values of LAI (2.55), light interception (33.63\%), and SPAD meter reading $(22.10 \%)$. These results are in agreement with the findings of Biddington and Dearman (1985), who reported that mechanically induced stress (MIS) analogous to artificial lodging facilitated greater release of ethylene which induced higher growth responses, like LAI, LAD, light interception and higher SPAD meter readings.

\section{Effect on yield parameters}

The grain yield is an end product, which obviously depends upon total dry matter production per plant at different stages of crop growth and its higher apportioning into the reproductive parts and panicle length, number of grains and number of spikelets (Table 8). The results indicated that between degrees of artificial lodging, $45^{0}$ recorded significantly higher grain yield of $1987 \mathrm{~kg} \mathrm{ha}^{-1}$ compared to $90^{0}\left(1796 \mathrm{~kg} \mathrm{ha}^{-1}\right)$. Among different stages of artificial lodging, before heading stage being on par with conventional practice (nolodging) (2082 $\left.\mathrm{kg} \mathrm{ha}^{-1}\right)$ produced significantly higher grain yield $\left(2201 \mathrm{~kg} \mathrm{ha}^{-1}\right)$. Lodging at mealy ripe stage gave significantly lower grain yield of $1681 \mathrm{~kg} \mathrm{ha}^{-1}$ (Table 9).

The interactions between degree of lodging and stages of lodging revealed that the grain yield with $45^{\circ}$ compared to $90^{\circ}$ of artificial lodging was significantly higher at before 
heading $\left(2385 \mathrm{~kg} \mathrm{ha}^{-1}\right)$ and at hard kernel stage $\left(1987 \mathrm{~kg} \mathrm{ha}^{-1}\right)$, numerically higher at heading (2061 kg ha $\left.{ }^{-1}\right)$ and at conventional treatment $\left(2082 \mathrm{~kg} \mathrm{ha}^{-1}\right)$, respectively. At mealy stage of artificial lodging recorded significantly lower grain yield was observed $\left(1724 \mathrm{~kg} \mathrm{ha}^{-1}\right)$. The extent of its superiority ( $45^{0}$ lodging) compared to control at before heading was $14.6 \%$, at heading $-1.0 \%$ at mealy stage $-17.2 \%$ and at hard kernel stage $4.6 \%$. Comparing the interactions occurring between stages of bending and $90^{\circ}$ of lodging indicated that reduction in the grain yield compared to control at before heading was $3.2 \%$, at heading $-5.2 \%$, at mealy stage $21.4 \%$ and at hard kernel stage it was $-13.7 \%$. This may be attributed to significantly higher LAI (2.55), LAD (75.40 days), total dry matter accumulation (114.2 $\left.\mathrm{g} \mathrm{plant}^{-1}\right)$, light interception $(33.63 \%)$, Spad meter reading (37.11\% at initial flowering), number of spikelet's (16.97 panicle ${ }^{-1}$ ) and number of grains panicle ${ }^{-1}$ (34.84), thousand grain weight $(39.80 \mathrm{~g})$. On the other hand, $45^{0}$ of artificial lodging imposed at mealy ripe stage of artificial lodging recorded significantly lower LAI (2.55), light interception (24.63\%), spad meter reading $(22.10 \%)$, total dry mater accumulation $\left(82.07 \mathrm{~g}\right.$ plant $\left.^{-1}\right)$, number of productive tillers per meter row length (68.25) and per square meter area (273.34), number of spikelets panicle $^{-1}$ (16.57), number of grains panicle $^{-1}$ (33.50), panicle weight (61.08 g plant $\left.^{-1}\right)$ and thousand grain weight (35.44g).

In conclusion our results indicated that artificial lodging at $45^{\circ}$ compared to $90^{\circ}$ was recorded significantly higher grain yield at before heading ( $\left.2385 \mathrm{~kg} \mathrm{ha}^{-1}\right)$, at hard kennel stage $(1987 \mathrm{~kg} / \mathrm{ha})$ and at mealy ripe stage $\left(1724 \mathrm{~kg} \mathrm{ha}^{-1}\right)$ and numerically higher at heading $\left(2061 \mathrm{~kg} \mathrm{ha}^{-1}\right)$ and at conventional treatment $\left(2082 \mathrm{~kg} \mathrm{ha}^{-1}\right)$. Hence proper agronomic management strategies such as foliar application of growth regulators need to be adopted at the susceptible stages to prevent the detrimental effects of lodging and further losses on grain yield of dicoccum wheat of the region.

\section{References}

Baker, C.J., Berry, P.M., Spink, J.H., Sylvester-Bradley, R., Griffin, J.M., Scott, R.K. and Clare, R.W., 1998. A method for the assessment of the risk of wheat lodging. J. Theor. Biol., 194, 587-603.

Berry, P. M., Sterling, M., Spink, J. H., Baker, C. J., Sylvester- Bradley, R., Mooney, S. J., Tams, A. R. and Ennos, A. R., 2004, Understanding and reducing lodging in cereals. Adv. Agron., 84: 217-271.

Biddington, N. L. 1986. The effects of mechanically - induced stress in plants- a review, Plant growth regulation 4:103-123.

Biddington, N. L and Dearman, A. S. 1985. Stress induced effects in vegetable seedlings. Rep national vegetable Research Station, Wellesbourne Warwick CV 35 9EF for 1984:103104.

Davies P J, 2004. Introduction, In: Davies P. $\mathrm{J}$, ed, Plant Hormones, Biosynthesis, Signal Transduction, Action! Dordrecht, Kluwer Academic Publishers, The Nether lands. Pp. 1-35 Davies W, J, and Zhang J, H, 1991, Root Signals and the regulation of growth and development of plants in drying soil. Annual Review of Plant physiology and plant molecular Biology 42, 55-76.

Foulkes M. J, Slafer, G. A, Davies, W. J, Berry, P. M, Sylvester - Bradley R, Martre, P, Calderini D.F, Griffiths, S, and Reynolds, M.P, 2011. Raising yield potential of wheat III. Optimizing partitioning to grain while maintaining lodging resistance 
Journal of Experimental Botany, 62 (2): $469-486$.

Fischer R. A. and Stapper, M., 1987. Lodging effects on high - yielding crops of irrigated semi - dwarf wheat. Field Crops Research, 17 (3-4): 245-258.

Liu, X, Ju, X, Zhang. F, Pan, J., and Christie P., 2003. Nitrogen dynamics and budgets in a winter Wheat -Maize Cropping System in the North Chaina Plain, Field Crops Research, 83 (2): 111-124.

Pumphrey, P. V. and Rubenthaler, G. L, 1983. Lodging effects on yield and quality of soft white wheat. Cereal Chemistry, 60 (4): 268 - 270.

Yang Jian Chang, Zhang Hao and Zhang Jian, hua, 2012. Root morphology and Physiology in relation to the yield formation of Rice. Journal of integrative Agriculture, 11 (6): 920926.

Yang J. C. 2011. Relationship of rice root morphology and physiology with the formation of grain yield and quality and the nutrient absorption and utilization. Scientica Agricultura Sinica, 44: 33 - 46 (in Chinese).

Zahedi M. and Jenner C. F, 2003. Analysis of affects in Wheat of high temperature on grain filling attributes estimated from Mathematical Models of grain filling. Journal of Agricultural Science, 141: 203-212.

\section{How to cite this article:}

Sujatha M. Guled, B.N. Aravinda Kumar, B.T. Nadagouda, Ramesh Babu and Hunshal, C.S. 2018. Artificial Lodging: An Effort to Identify a Critical Crop Growth Stage Susceptible to Lodging Leading to Yield Loss and Modification of Yield Structure in Dicoccum Wheat. Int.J.Curr.Microbiol.App.Sci. 7(12): 2920-2932. doi: https://doi.org/10.20546/ijcmas.2018.712.333 